\title{
In-Situ TEM studies of Lithium Ion Battery
}

C. M. Wang ${ }^{*}$, W. Xu ${ }^{* *}$, B. W. Arey*, L. V. Saraf*, D. W. Choi**, J. Liu ${ }^{* * *}$, Z. G. Yang ${ }^{* *}$, J. G. Zhang ${ }^{* *}$, S. Thevuthasan ${ }^{*}$, and D. R. Baer ${ }^{*}$

* Environmental Molecular Sciences Laboratory,

*** Energy and Environmental Directorate

**** Fundamental and Computational Science Directorate

Pacific Northwest National Laboratory, Richland, WA 99352 USA

Li-ion batteries, as similarly with other electrochemical energy storage devices, are complex multi-component systems that incorporate widely dissimilar phases in physical and electrical contact [1]. Repeated charging and discharging of the Li-ion battery induces microstructural evolutions both at the interface between the electrolyte and the electrode and within the electrode. Although it has been established that this microstructural evolution is responsible for the failure of the battery, the mechanisms of the microstructural changes as a function of charging/discharging are not well understood.

Transmission electron microscopy (TEM) and spectroscopy have been evolved to a stage such that it can be routinely used to probe into both the structural and chemical composition of the materials with a spatial resolution of a single atomic column, a direct in-situ TEM observation of structural evolution of the materials in lithium ion battery during the dynamic operation of the battery has never been reported. This is related to three factors: high vacuum operation of a TEM; electron transparency requirement of the region to be observed, and the difficulties dealing with the liquid electrolyte of lithium ion battery. In this paper, we report the results of exploring the in-situ TEM techniques for observation of the interface in lithium ion battery during the operation of the battery. The objective of this paper is to explore the possibilities of studying the structure and chemical composition of the interface across the anode and the electrolyte in a lithium ion battery during the dynamic operation of the battery using TEM imaging, electron diffraction, and electron energy loss spectroscopy.

A miniature prototype battery was built using $\mathrm{LiCoO}_{2}$ as the cathode, $\mathrm{SnO}_{2}$ nanowire as anode and an ionic liquid electrolyte. The cathode was a mixture of $\mathrm{LiCoO}_{2}$ particles, conductive carbon and PVDF binder, and coated as a thin film on an aluminum foil. The anode was $\mathrm{SnO}_{2}$ nanowire, which gives the advantage of electron transparency and therefore allows in-situ observation of both chemical and structural change under TEM during the operation of the battery. The electrolyte was air stable where the salt lithium bis(trifluoromethane sulfonyl)imide (LiTFSI) was dissolved in a hydrophobic ionic liquid 1-butyl-1-methylpyrrolidium $\left(\mathrm{P}_{14}\right)$ bis(trifluoromethane sulfonyl)imide $\left(\mathrm{P}_{14} \mathrm{TFSI}\right)$. The overall composition of the electrolyte was $10 \%$ LiTFSI in $\mathrm{P}_{14}$ TFSI. It has been tested that this ionic-liquid-based electrolyte is stable in the column of the TEM with a typical vacuum of $10^{-5} \mathrm{~Pa}$ and shows no significant evaporation following one week in the TEM column, therefore allowing a systematic cyclic charging and discharging of the battery in the TEM [2].

During the initial charging, the cell potential as a function of charging time is shown in Figure 1. The structural of $\mathrm{SnO}_{2}$ anode at a specific site was periodically observed using TEM imaging and electron diffraction, which is overlaid on the initial charging curve. 
The TEM images reveal that during the initial charging a thin layer of amorphous materials gradually accumulate on the surface of the nanowire as indicated by the arrows in Figure 1. Electron diffraction analysis reveals that the anode shows no detectable changes with the progress of the initial charging, even the potential of the cell is gradually increasing. It is also noticed that on the charging curve, there are some cusps at which the cell potential drops. The arising of the cusps on the charging curve is related to the electron beam of the TEM for imaging or diffraction analysis.

In this work we have demonstrated some basic design concept for in-situ TEM observation of the microstructural evolution of the lithium ion battery. For the case of a battery using $\mathrm{SnO}_{2}$ anode, ionic-liquid-based electrolyte and $\mathrm{LiCoO}_{2}$ cathode, decomposition of the electrolyte and subsequent electrodeposition on the anode leads to the formation of a coating layer on the anode. The coated layer is enriched with $\mathrm{Li}$. Formation of the coating layer on the $\mathrm{SnO}_{2}$ anode prevents the $\mathrm{Li}$ intercalation into the anode, which accounts for the observed low capacity and fast fading of the Li-ion coin cell using this type of ionic liquid electrolyte.

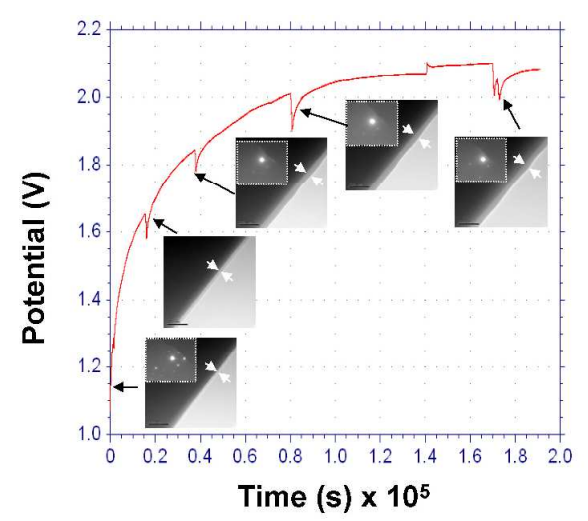

Fig. 1. The cell potential as a function charging time during the initial charging. The TEM images and the corresponding diffraction patterns indicate the possible change of the nanowire at each stage of charging. Note the increasing thickness of the surface coating layer on the nanowire with the progression of the charging. Diffraction shows no obvious change in the nanowire structure. The cusps on the charging curve are related to the electron beam effect on the cell during the TEM imaging.

Acknowledgements: This work was supported U.S. Department of Energy (DOE) Office of Science, Offices of Basic Energy Sciences and Biological and Environmental Research. The work was conducted in the William R. Wiley Environmental Molecular Sciences Laboratory (EMSL) a DOE User Facility operated by Battelle for the DOE Office of Biological and Environmental Research. Pacific Northwest National Laboratory is operated for the DOE under Contract DE-AC06-76RLO 1830.

\section{References}

[1] J. M. Tarascon and M. Armand, Nature 414 (2001) 359.

[2] C. M. Wang, W. Xu, J. Liu, D. W. Choi, B. W. Arey, L. V. Saraf, J. G. Zhang, Z. G. Yang, S. Thevuthasan, D. R. Baer, and N. Salmon, Nanotechnology, under review. 